\title{
Discovery and validation of a glioblastoma co-expressed gene module
}

\author{
Leland J. Dunwoodie ${ }^{1}$, William L. Poehlman ${ }^{1}$, Stephen P. Ficklin² and Frank \\ Alexander Feltus ${ }^{1}$ \\ ${ }^{1}$ Department of Genetics and Biochemistry, Clemson University, Clemson, SC 29634, USA \\ ${ }^{2}$ Department of Horticulture, Washington State University, Pullman, WA 99164, USA \\ Correspondence to: Frank Alexander Feltus, email: ffeltus@clemson.edu
}

Keywords: glioblastoma; systems biology; gene co-expression networks; complement system; cancer

Received: July 28, $2017 \quad$ Accepted: January 09, $2018 \quad$ Published: January 13, 2018

Copyright: Dunwoodie et al. This is an open-access article distributed under the terms of the Creative Commons Attribution License 3.0 (CC BY 3.0), which permits unrestricted use, distribution, and reproduction in any medium, provided the original author and source are credited.

\section{ABSTRACT}

\begin{abstract}
Tumors exhibit complex patterns of aberrant gene expression. Using a knowledge-independent, noise-reducing gene co-expression network construction software called KINC, we created multiple RNAseq-based gene co-expression networks relevant to brain and glioblastoma biology. In this report, we describe the discovery and validation of a glioblastoma-specific gene module that contains 22 co-expressed genes. The genes are upregulated in glioblastoma relative to normal brain and lower grade glioma samples; they are also hypo-methylated in glioblastoma relative to lower grade glioma tumors. Among the proneural, neural, mesenchymal, and classical glioblastoma subtypes, these genes are most-highly expressed in the mesenchymal subtype. Furthermore, high expression of these genes is associated with decreased survival across each glioblastoma subtype. These genes are of interest to glioblastoma biology and our gene interaction discovery and validation workflow can be used to discover and validate co-expressed gene modules derived from any co-expression network.
\end{abstract}

\section{INTRODUCTION}

Glioblastoma (GBM) tumors, with an adult median survival time of 14.6 months after radiation and temozolomide therapy [1], are known for their heterogeneity, vascularization, and lethality. Even after resection, remaining tumor cells multiply and invade the surrounding parenchyma. Interestingly, primary GBM has few known risk factors [2] -- GBM affects patients across age, cultural, and socioeconomic boundaries. The discovery of relevant biomarker combinations driving GBM tumorgenicity would have therapeutic implications.

There are known monogenic GBM biomarkers that include mutations in the IDH1 [3] and PDGFR $\alpha$ [4] loci. However, any given biomarker does not provide a complete picture of the GBM microenvironment. GBM tumors, as with other tumors, diseases, and complex traits, are controlled by a variety of genetic and epigenetic factors [5]. Thus, a systems approach is needed to fully understand the biology underlying the GBM phenotype.
Fortunately, modern measurement technologies such as next-generation sequencing [6] now provide researchers a broad genomics perspective that is revealing new insights to human disease. These technologies, coupled with genomics and epigenomics databases such as The Cancer Genome Atlas (TCGA) [7], used in this investigation, encourage new discoveries.

To identify complex gene expression relationships in multiple human tumors, we have built RNAseq-based gene expression matrices (GEMs) from publicly available RNAseq datasets. One GEM contains gene expression profiles for GBM, lower grade glioma (LGG), bladder urothelial carcinoma (BLCA), thyroid carcinoma (THCA), and ovarian serous cystadenocarcinoma (OV) from TCGA [7] and is fully described in another publication [8]. Another GEM built for this study contains different RNAseq expression profiles for GBM [9], normal brain [10], and Parkinson's brain [11] obtained from the NCBI SRA archive [12]. Both GEMs were individually preprocessed and transformed into a gene co-expression 
network (GCN) using Knowledge-Independent Network Construction (KINC) software [8]. Complex gene interactions present in the input samples are mineable from these GCNs, and these gene interactions patterns can be compared between GCNs.

The KINC software package ([8]; open source code available at http://www.github.com/SystemsGenetics/ $\mathrm{KINC}$ ) is unique because it deconvolutes mixed-condition expression patterns (e.g. mixed tumor expression profiles in the GEM), allowing significant co-expression relationships to be annotated with sample labels (e.g. GBM vs. non-GBM). There is no need to separate gene expression profiles prior to analysis. Thus, without providing KINC a priori knowledge, tumor- or non-tumorspecific gene expression relationships can be identified and analyzed for biological meaning.

In this report, we apply a generalizable gene interaction discovery and validation workflow, outlined in Supplementary Figure 1, that allows for the detection of condition-specific gene sets in one GCN that can be validated for specificity and reproducibility in alternate GCNs. We focused this approach on GBM-specific modules to investigate GBM tumor biology. Herein, we describe the discovery and characteristics of a GBMspecific gene module present in two GCNs, and explore the expression patterns and epigenetic state of these genes across the spectrum of GBM subtypes using multiple in silico approaches.

\section{RESULTS AND DISCUSSION}

\section{GCN construction}

KINC identified significant co-expression relationships among 2016 datasets in the TCGA Network and among 204 datasets in the Brain Network. The TCGA network, obtained from our previous study in Ficklin et al. [8], included GBM and LGG datasets along with BLCA, $\mathrm{OV}$, and THCA datasets. The Brain Network included GBM and normal brain datasets along with datasets from Brodmann's Area 9 of Parkinson's patients [11]. The TCGA Network (Supplementary Table 1) is described in [8] and the Brain Network (Supplementary Table 2) was visualized with Cytoscape [41] and shown in Figure 1. 356 LCMs were detected in the TCGA Network ([8]; Supplementary Table 3) and 456 LCMs were found in the Brain Network (Supplementary Table 4). Many of these modules were condition-specific; for example, 68 of the Brain Network's 456 modules were enriched for GBM $(p<0.001)$.

\section{Gene module discovery}

Genes in the Brain Network were compared with those in the TCGA Network to investigate commonality in GBM co-expression. 477 unique genes -- 6.6\% of the unique genes in the TCGA Network and $8.9 \%$ of the unique genes in the Brain Network-were found in both networks. In addition, each network was parsed into LCMs. The TCGA Network had 356 modules total and the Brain Network had 456 modules total. Considering only the genes in these modules, 74 unique genes mapped between networks. The low amount of overlap may be a function of (A) sparse gene overlap between GCNs; and (B) differential Type I and Type II error in each GCN partially due to alternate expression measurement techniques; the TCGA Network used a GEM made with TCGA's RNAseqV2 workflow [15] and 73599 knownGene 5 UCSC transcript IDs, while the Brain Network used a GEM made with Hisat [18], Cufflinks [21], and 209086 Ensembl hg38 transcript IDs. Nonetheless, of these 74 overlapping genes, 22 (Table 1) were seen in Modules M0257 (in the Brain Network) and M0214 (in the TCGA Network). While the fate of other common interactions is unclear, these 22 intersecting GBM interactions emerged in both GCNs.

Next, we asked if there was corroborative coexpression evidence for the 22 interacting genes from other sources. Specifically, we searched the 22 matching genes as a group in the GeneFriends gene co-expression database [27]. Table 1 shows the gene set co-expression analysis where co-expression probability is represented by the GeneFriends binomial cumulative distribution function $(p<1.00 \mathrm{E}-7)$. Because 22 genes were provided to GeneFriends, each gene has a maximum of 22 friends, or co-expressed genes.

Interestingly, TCGA M0214 $(p<2.56 \mathrm{E}-17)$ and Brain M0257 $(p<1.37 \mathrm{E}-15)$ were both enriched for GBM but not LGG $(p<4.52 \mathrm{E}-03$ in the TCGA Network) or normal brain ( $p<1.00$ in the Brain Network). TCGA M0214 is also enriched for ovarian cancer (OV; $p<2.56 \mathrm{E}-17)$. Table 2 shows the condition-specific Fisher's Exact Test enrichment values for TCGA M0214 and Brain M0257. In total, TCGA M0214 includes 54 genes and Brain M0257 includes 63 genes (Supplementary Table 5). The clinical annotation term enrichment results are available for each TCGA module (Supplementary Table 3) and each Brain module (Supplementary Table 4).

It was interesting that TCGA M0214 is enriched for both GBM and OV. While OV is enriched for 57 modules and GBM is enriched for 102 modules (Supplementary Table 3), 22 modules are enriched for both GBM and OV. While these cancers are seemingly very different, further investigations might reveal new commonalities between them. Indeed, the literature shows few links between GBM and OV, but OV can metastasize to the brain [42] and bevacizumab, an angiogenesis inhibitor used to treat GBM, has been found to alleviate mesenchymal-like, proliferative $\mathrm{OV}$ subtypes [43]. While TCGA M0282, described in Ficklin et al. [8], is also enriched for GBM and OV in addition to THCA, no genes are shared between TCGA M0282 and M0257. Indeed, no genes are shared between TCGA M0282 and the Brain Network. 


\section{Internetwork gene module validation shows GBM-specific correlations}

The correlations in Brain M0257, which is enriched for GBM, were compared to matching correlations found by KINC using only the normal brain datasets in the Brain GEM. 70 of the 154 edges (45.45\%) in Brain M0257 were rediscovered in the normal brain datasets. Of these edges, none had a significant Spearman correlation greater than 0.8801, the Brain Network's RMT threshold (Figure 2). TCGA M0214 includes 54 unique transcripts and 416 edges correlated above its RMT threshold. 49 of these UCSC kg5 transcripts mapped to the hg38 Ensembl IDs used by the Brain and Random GEMs. As described in the Methods section, the expression values for these 49 transcripts in the Random GEM were processed with KINC [8]. KINC rediscovered 191 (45.91\%) of the 416 TCGA edges using expression values from the Random GEM. Nine $(4.71 \%)$ of the 191 rediscovered edges in the Random GEM had a Spearman correlation greater than 0.7901, the Random Network's RMT threshold. Seven (4.55\%) of the 154 edges in Brain M0257 were also found in TCGA M0214; $100 \%$ of these edges have a Spearman correlation greater than the Brain Network's significance threshold (Figure 3). These data indicate that the pairwise correlation of these genes is GBM-specific.

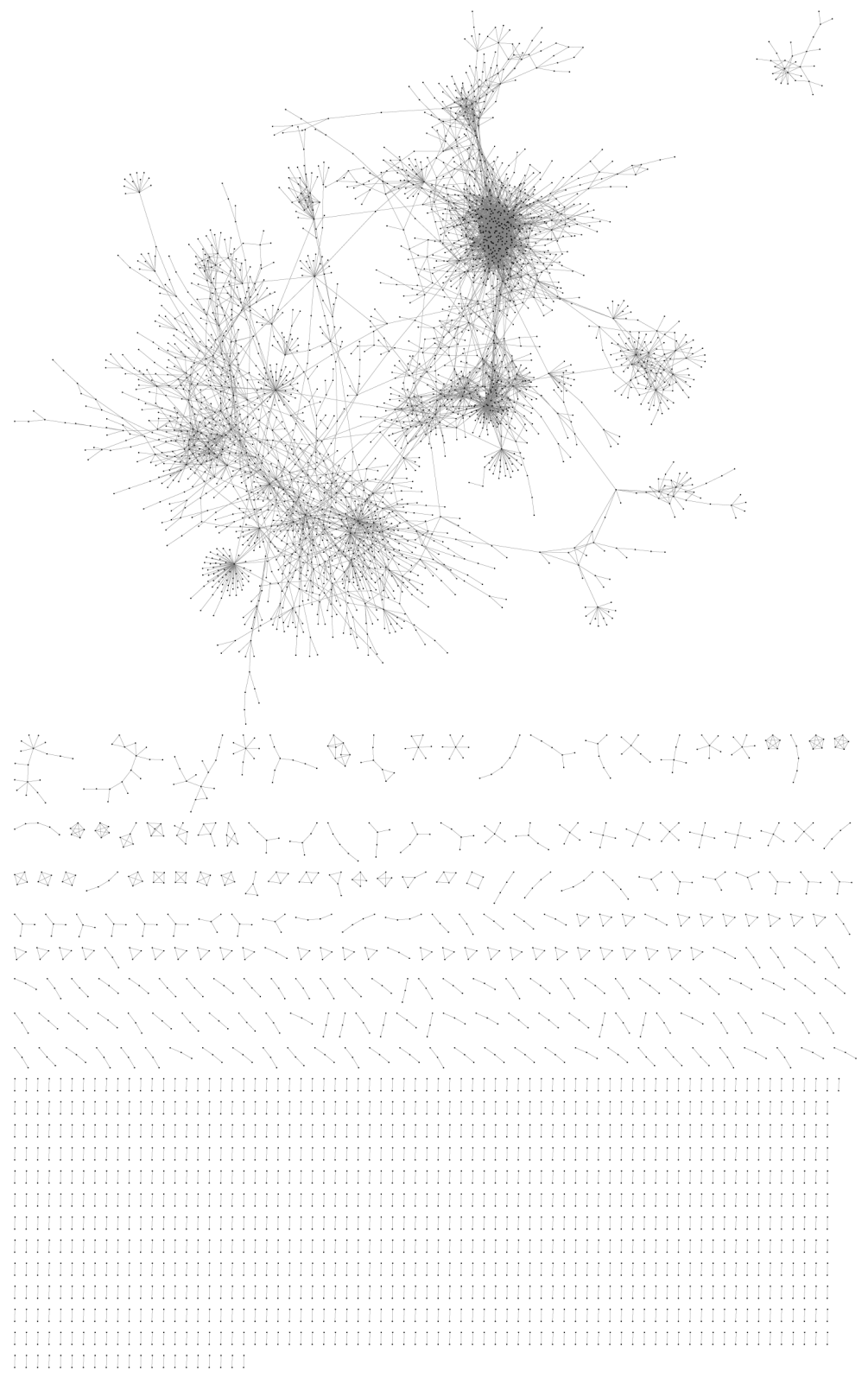

Figure 1: Visualization of the brain network. Points represent transcripts (nodes) and lines represent significant expression correlations (edges) between nodes. 
Table 1: The 22 genes overlapping between TCGA M0214 and brain M0257

\begin{tabular}{|c|c|c|c|c|c|c|c|c|}
\hline $\begin{array}{l}\text { Gene } \\
\text { Symbol }\end{array}$ & Gene Name & hg38 Ensembl ID & kg5 UCSC ID & $\begin{array}{c}\text { Entrez } \\
\text { ID }\end{array}$ & Chromosome & $\begin{array}{c}\text { Transcription } \\
\text { Start }\end{array}$ & $\begin{array}{c}\text { Transcription } \\
\text { Stop }\end{array}$ & Gene Friends \\
\hline LAPTM5 & $\begin{array}{l}\text { lysosomal protein } \\
\text { transmembrane } 5\end{array}$ & ENST00000294507 & uc002iop. 1 & 7805 & 1 & 30732469 & 30757820 & 19 \\
\hline C1QA & $\begin{array}{l}\text { complement } \mathrm{C} 1 \mathrm{q} A \\
\text { chain }\end{array}$ & ENST00000374642 & uc001bfy. 2 & 712 & 1 & 22636506 & 22639608 & 10 \\
\hline FCER1G & $\begin{array}{l}\text { Fc fragment of } \operatorname{IgE} \\
\text { receptor Ig }\end{array}$ & ENST00000367992 & uc001bga. 3 & 2207 & 1 & 161215279 & 161220699 & 22 \\
\hline C1QC & $\begin{array}{l}\text { complement } \mathrm{C} 1 \mathrm{q} \mathrm{C} \\
\text { chain }\end{array}$ & ENST00000374639 & uc001qtv.1 & 714 & 1 & 22643633 & 22648110 & 11 \\
\hline CD86 & CD86 molecule & ENST00000330540 & uc002jkv.2 & 942 & 3 & 122055374 & 122121136 & 22 \\
\hline $\begin{array}{l}\text { HAVCR2 } \\
(\text { TIM-3) [23] }\end{array}$ & $\begin{array}{l}\text { hepatitis A virus } \\
\text { cellular receptor } 2\end{array}$ & ENST00000307851 & uc003eet. 2 & 84868 & 5 & 157085832 & 157109714 & 21 \\
\hline LY86 & lymphocyte antigen 86 & ENST00000379953 & uc001 fyz.1 & 9450 & 6 & 6588108 & 6654983 & 16 \\
\hline TREM2 & $\begin{array}{l}\text { triggering receptor } \\
\text { expressed on myeloid } \\
\text { cells } 2\end{array}$ & ENST00000373113 & uc001nym.2 & 54209 & 6 & 41158507 & 41163176 & 9 \\
\hline FERMT3 & $\begin{array}{l}\text { fermitin family member } \\
\qquad 3\end{array}$ & ENST00000345728 & uc001xvv. 2 & 83706 & 11 & 64206734 & 64223886 & 19 \\
\hline SPI1 & Spi-1 proto-oncogene & ENST00000378538 & uc0031wk.1 & 6688 & 11 & 47354860 & 47378576 & 19 \\
\hline C3AR1 & $\begin{array}{l}\text { complement } \mathrm{C} 3 \mathrm{a} \\
\text { receptor } 1\end{array}$ & ENST00000307637 & uc002zgf.3 & 719 & 12 & 8058302 & 8066471 & 21 \\
\hline GPR65 & $\begin{array}{l}\text { G protein-coupled } \\
\text { receptor } 65\end{array}$ & ENST00000267549 & uc001bsc. 2 & 8477 & 14 & 88005124 & 88014811 & 17 \\
\hline RNASE6 & $\begin{array}{l}\text { ribonuclease A family } \\
\text { member k6 }\end{array}$ & ENST00000304677 & uc003mwy.1 & 10048 & 14 & 20781051 & 20782467 & 22 \\
\hline $\mathrm{ABI} 3$ & ABI family member 3 & ENST00000225941 & uc002mkg.2 & 51225 & 17 & 49210227 & 49223225 & 21 \\
\hline CD300A & CD300a molecule & ENST00000360141 & uc011aqf.1 & 11314 & 17 & 74466416 & 74484796 & 19 \\
\hline TYROBP & $\begin{array}{l}\text { TYRO protein tyrosine } \\
\text { kinase binding protein }\end{array}$ & ENST00000262629 & uc001vye. 3 & 7305 & 19 & 35904410 & 35908295 & 22 \\
\hline SIGLEC9 & $\begin{array}{l}\text { sialic acid binding Ig } \\
\text { like lectin } 9\end{array}$ & ENST00000250360 & uc004euu.2 & 27180 & 19 & 51124908 & 51130310 & 19 \\
\hline MYO1F & myosin IF & ENST00000613525 & uc002pvu. 2 & 4542 & 19 & 8520797 & 8577577 & 18 \\
\hline ITGB2 & integrin subunit beta 2 & ENST00000397852 & uc001nfb.1 & 3689 & 21 & 44885953 & 44910826 & 22 \\
\hline PARVG & parvin gamma & ENST00000356909 & uc003opy.2 & 64098 & 22 & 44181400 & 44206635 & 19 \\
\hline WAS & $\begin{array}{l}\text { Wiskott-Aldrich } \\
\text { syndrome }\end{array}$ & ENST00000376701 & uc002ocm. 2 & 12731 & $\mathrm{X}$ & 48683779 & 48691427 & 19 \\
\hline SASH3 & $\begin{array}{l}\text { SAM and SH3 domain } \\
\text { containing } 3\end{array}$ & ENST00000356892 & uc004dkm.3 & 54440 & $\mathrm{X}$ & 129779984 & 129795201 & 19 \\
\hline
\end{tabular}

\section{Gene expression analysis reveals GBM-specific upregulation}

We investigated the gene expression levels of the 22 genes seen in TCGA M0214 and Brain M0257 in different conditions. Heatmaps and bar graphs were constructed to visualize the expression levels of the 22 matching genes between TCGA M0214 and Brain M0257 (Figures 4A-4B and 5A-5B). All 22 genes showed significantly upregulated expression (Student's $T$ Test; $p<0.001$ ) in GBM relative to LGG (in the TCGA Network) and relative to normal brain (in the Brain Network). Significance test results are available for the expression of the 22 shared genes in the TCGA GEM (Supplementary Table 6) and in the Brain GEM (Supplementary Table 7).

\section{Putative transcriptional regulators of the GBM modules}

Several gbmSygnal biclusters [37] involving genes from TCGA M0214 or Brain M0257 (Supplementary Table 8) implied regulation by the transcription factor ELF1. In addition, by loading the 22 matching genes into the Broad Institute Gene Set Enrichment Analysis [34], we found that ELF1 was the second-most enriched transcription factor for this dataset $\left(p<9.91 \times 10^{-8}\right)$ (Supplementary Table 9). Indeed, 5 of the 22 matching genes have putative ELF1 sites. Moreover, one ELF1 transcript, ENST00000239882, was upregulated in the Brain GEM GBM data relative to normal brain data $\left(p<3.79 \times 10^{-27}\right)$ (Supplementary Table 10). Furthermore, 
this transcript's kg5 UCSC counterpart, uc001uxs.2, was also upregulated in the TCGA GEM GBM data relative to LGG data $\left(p<1.94 \times 10^{-4}\right)$ (Supplementary Table 10).

In addition, one of the 22 matching genes, SPI1, is a transcription factor that shares the ETS transcription factor family with ELF1. The 22 matching genes were also provided to the transcription factor function in the GeneFriends database [27] (Supplementary Table 11). Of 1538 possible transcription factors, SPI1 was the 5thmost enriched for the gene set $\left(p<1.01 \times 10^{-23}\right)$ and 19 of the 22 genes were co-expressed with SPI1. In addition, the 22 matching genes were provided to the RegNetwork database [35] (Supplementary Table 12). SPI1 was the only one of the 22 matching genes to be considered a regulator by RegNetwork. Eight of the 21 other genes, three with high confidence, were considered regulated by SPI1. Indeed, of the 2221 genes potentially regulated by SPI1, three of the 22 genes ranked very highly-ITGB2 was ranked 3rd, WAS 5th, and TYROBP 27th.

\section{Internetwork modular methylation analysis shows GBM-specific hypo-methylation}

The beta methylation values for each of the 22 matching genes were evaluated using data from TCGA. A Student's $T$-Test was used to compare the beta methylation values for LGG with those in GBM $\left(p<6.84 \times 10^{-6}\right)$. As shown in Figure 6, on average, each of the 22 genes was hypo-methylated in GBM relative to LGG. Of interest, while TCGA M0214 is enriched for GBM and OV, the methylation patterns differ between GBM and OV. While these 22 genes are hypo-methylated in GBM versus LGG datasets, several of these 22 genes are hyper-methylated in
OV versus LGG datasets (data not shown), suggesting an alternate regulatory mechanism in $\mathrm{OV}$.

\section{GBM subtype analysis supports the mesenchymal phenotype}

The gbmSygnal Network [37] uses bicluster technology to group genes based on ChIPseq signals and gene co-expression. Each of the 22 matching genes was searched in the gbmSygnal database. 16 biclusters enriched for a cancer hallmark were found with three or more of the 22 matching genes. These 16 biclusters were all enriched for "tumor-promoting inflammation" and "evading immune detection" (Supplementary Table 8). In addition, the gbmSygnal Network organizes expression data for each bicluster into quintiles and enriches each quintile for GBM subtype. Mesenchymal GBM was predominant in the highest expression quintile relative to the lowest expression quintile in each of the 16 biclusters. Furthermore, Verhaak et al. [44] described four subtypes of GBM [44] and genes upregulated in each of the four GBM subtypes. Four genes (SIGLEC9, MYO1F, LAPTM5, ITGB2) from the list of 22 matching genes were upregulated in mesenchymal GBM; none of the 22 genes were upregulated in any other subtype. Furthermore, we investigated the expression levels of nine NF1 transcripts in the Brain GEM. High NF1 expression is characteristic of mesenchymal GBM [44]. One of these transcripts, ENST00000358273, was upregulated in GBM relative to normal brain (Student's $T$-Test $p$-value $=6.56 \times 10^{-15}$; Supplementary Table 13). Finally, 17 of the 22 shared genes were found in the Glioblastoma Bio Discovery Portal [38] based on results from Verhaak

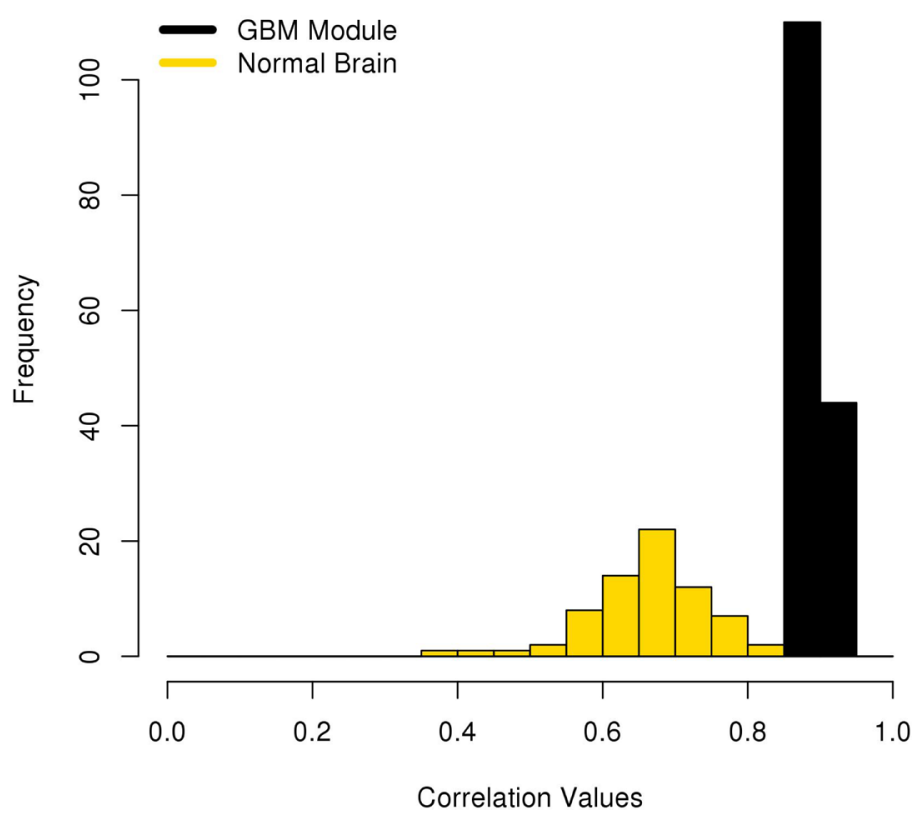

Figure 2: Brain M0257 Correlation values mapped to normal brain datasets. The Spearman correlation values of pairwise gene expression are shown for Brain M0257 and matching edges using only normal samples from the Brain GEM. 
Table 2: Condition-specific module enrichment

\begin{tabular}{lccc}
\hline Network & Module & Condition & Enrichment \\
\hline TCGA & M0214 & GBM & $2.56 \mathrm{E}-17$ \\
TCGA & M0214 & LGG & $4.52 \mathrm{E}-03$ \\
TCGA & M0214 & OV & $1.61 \mathrm{E}-14$ \\
TCGA & M0214 & THCA & $1.00 \mathrm{E}+00$ \\
TCGA & M0214 & BLCA & $1.00 \mathrm{E}+00$ \\
Brain & M0257 & GBM & $1.37 \mathrm{E}-15$ \\
Brain & M0257 & Normal Brain & $1.00 \mathrm{E}+00$ \\
Brain & M0257 & Parkinson & $2.05 \mathrm{E}-01$ \\
\hline
\end{tabular}

Table 3: Glioblastoma bio discovery portal survival analysis

\begin{tabular}{lcc}
\hline Subtype & Prognostic index hazard ratio & LogRank P-Value \\
\hline Classical & 3.57 & 0 \\
Neural & 15.63 & 0 \\
Proneural & 4.66 & 0 \\
Mesenchymal & 3.02 & 0 \\
Full Cohort & 1.79 & 0 \\
\hline
\end{tabular}

Prognostic index hazard ratio and logrank $p$-value are given for expression levels above the median within each subtype.

\section{Cancer and Random}

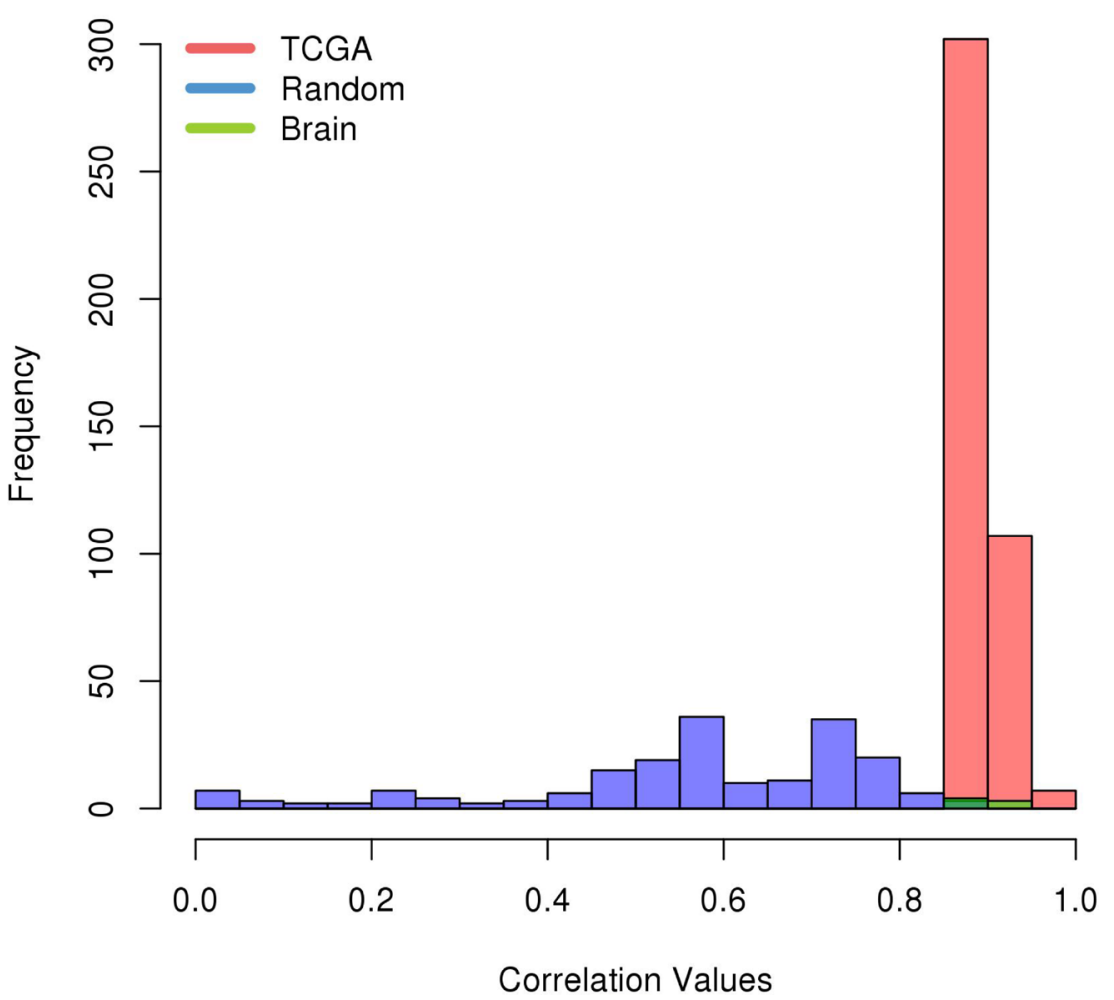

Figure 3: Correlation values from TCGA M0214 mapped to Brain M0257 and Random GEMs. The Spearman correlation values of pairwise gene expression are shown for TCGA M0214, Brain M0257, and a miniature GEM created using expression levels from the Random GEM and the genes in TCGA M0214. Only the edges in Brain M0257 that exactly match edges in TCGA M0214 are shown. 
et al. [44]. The average mRNA expression z-score was found across the proneural ( $n=56$ tumors), neural ( $n=31$ tumors), mesenchymal ( $n=57$ tumors), and classical ( $n=53$ tumors) GBM subtypes (Figure 7). The mesenchymal subtype showed the highest expression for 15 of 17 genes. Furthermore, using the Glioblastoma Bio Discovery Portal, it was found that above-median expression levels for these 17 genes led to decreased survival in every GBM subtype and the full cohort (Table 3).

\section{cBioPortal analysis provides evidence for a GBM-specific module}

If this 22-gene network is GBM-specific, as shown in Figures 4B and 5B, one would expect different cooccurrence and mutual exclusivity results for LGG and GBM data (Table 4). Indeed, this 22-gene network appears highly dependent on ATRX and p53 in LGG but not in GBM. The mutual exclusivity of PIK3R1 mutations in

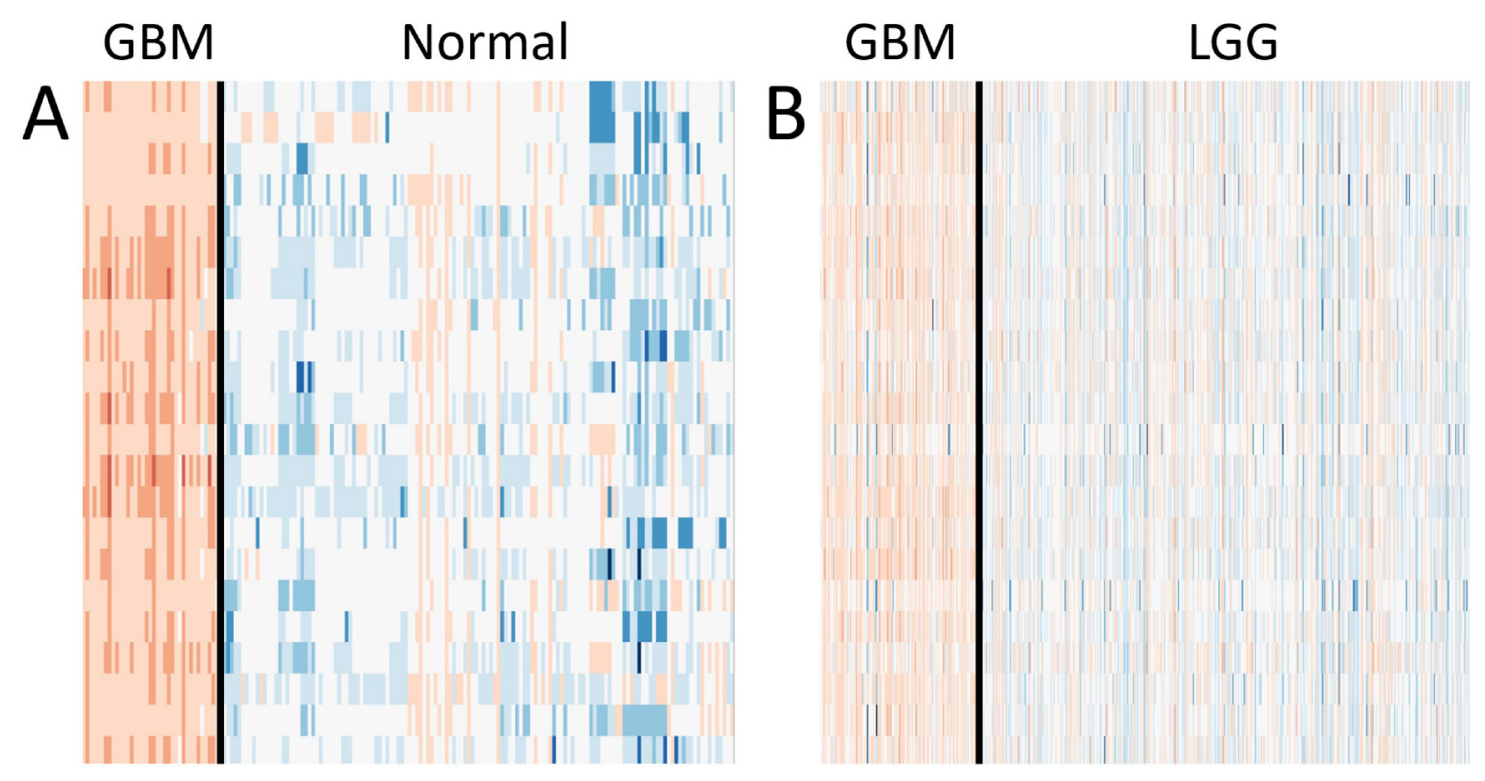

Figure 4: Expression levels of the 22 matching genes. (A) GBM and normal brain expression levels in the normalized Brain GEM. (B) GBM and LGG expression levels in the normalized TCGA GEM. Red indicates expression above the mean, blue indicates expression below the mean, and white indicates expression near the mean.
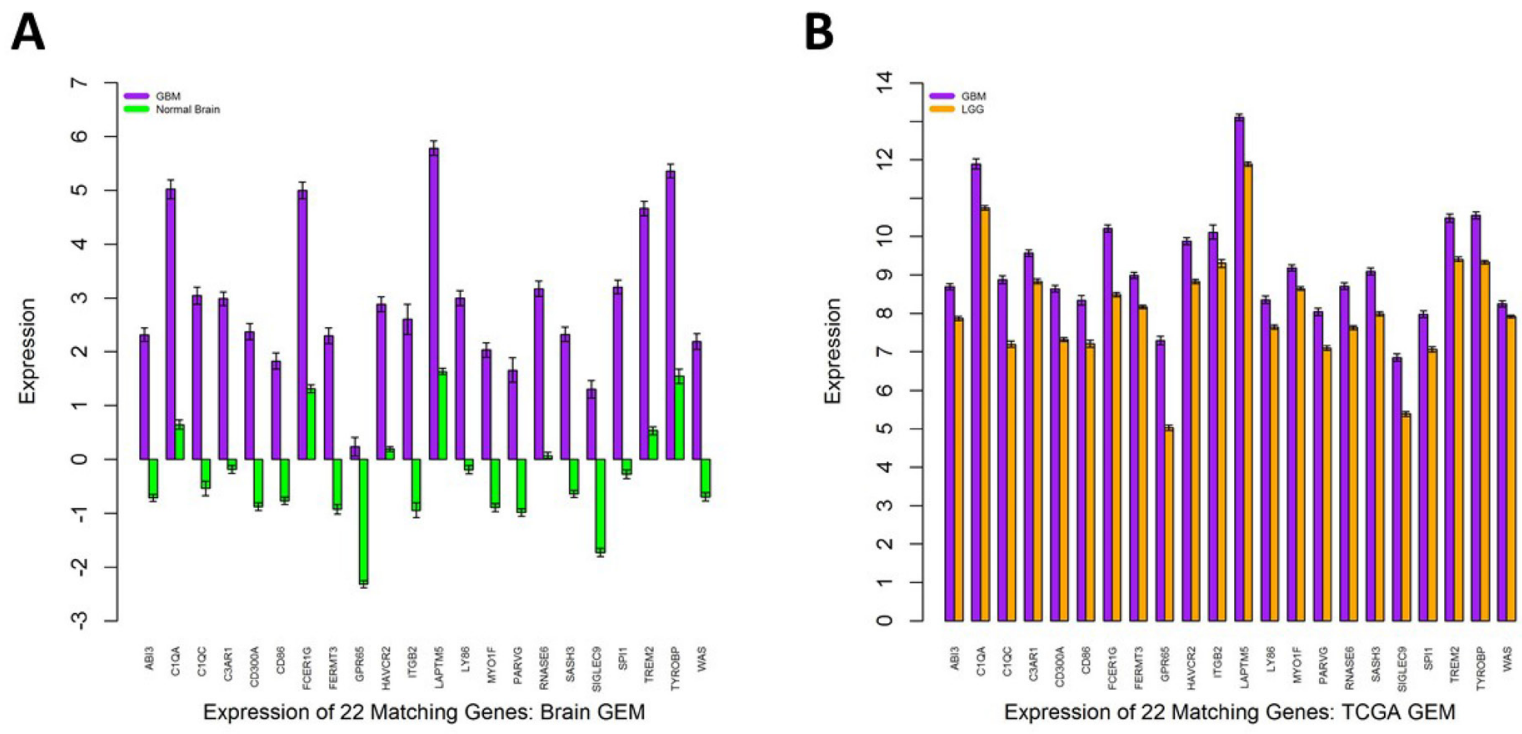

Figure 5: Expression levels of the 22 matching glioblastoma genes. (A) The expression of the 22 matching genes in the Brain GEM after dividing the normalized expression values by either the GBM or normal brain median expression value. Vertical axis units represent normalized FPKM values. (B) The expression of the 22 matching genes in the TCGA GEM. No further normalization was performed. Vertical axis units represent normalized RPKM values. Error bars represent SEM. 
GBM is of particular interest; PIK3R1 knockdown decreases invasion, proliferation, and migration in GBM [45]. IDH1 mutations were neither mutually exclusive nor co-occurring with alterations in the 22 shared genes. This is consistent with Figure 7; the proneural GBM subtype, which exhibits low expression for these 22 genes, is often defined by IDH1 or PDGFRA mutation.

\section{Internetwork modular functional annotation analysis}

We next sought to understand the function of TCGA M0214 and Brain M0257 in GBM. Comparing the functional annotations enriched $(p<0.001)$ in TCGA M0214 and Brain M0257 showed that 23 annotations were shared between modules (Table 5). C1QA and C1QC, components of complement protein $\mathrm{C} 1$ [46], and C3AR1, a receptor for the complement protein $\mathrm{C} 3 \mathrm{a}$ [47], are among the 22 shared genes between TCGA M0214 and Brain M0257. C1Q has been shown [48] to promote GBM invasiveness and proliferation independent of complement system activation. Carro et al. also lists $\mathrm{C} 1 \mathrm{Q}$ as a member of the transcriptional network which drives mesenchymal phenotypes in brain tumors [49]. Several complement system-related functional annotations (Table 5) are also shared between TCGA M0214 and Brain M0257. In addition, RNase6, one of the 22 matching genes, was searched with ImmuNet, a regulatory network database for immune system-related genes [36]. At a confidence level $>99 \%$, eight of the other 21 genes shared a function with RNase6 in the complement system (Supplementary Table 14).

In conclusion, we used a GMM-based gene co-expression analysis to identify GBM-specific gene co-expression clusters embedded within and parsed from LGG and normal brain datasets. A 22-gene module was separately identified in two gene expression sources; this module has increased RNA expression and decreased DNA methylation in GBM. Furthermore, high expression of these genes is associated with decreased survival and with the mesenchymal GBM subtype. Future work involving these genes may help assess their roles in the complex GBM phenotype. We present this GBM-specific gene module and note that the cross-validating co-expression workflow used here is widely applicable.

\section{MATERIALS AND METHODS}

\section{TCGA GEM construction}

As described in Ficklin et al. [8], the TCGA GEM was constructed using 2016 RNAseq tumor samples [7]. All available normalized isoform datasets, produced by TCGA's RNASeqV2 workflow [14, 15], were downloaded for five cancers on April 1, 2016. The datasets include 173 GBM samples, 534 lower grade glioma (LGG) samples,

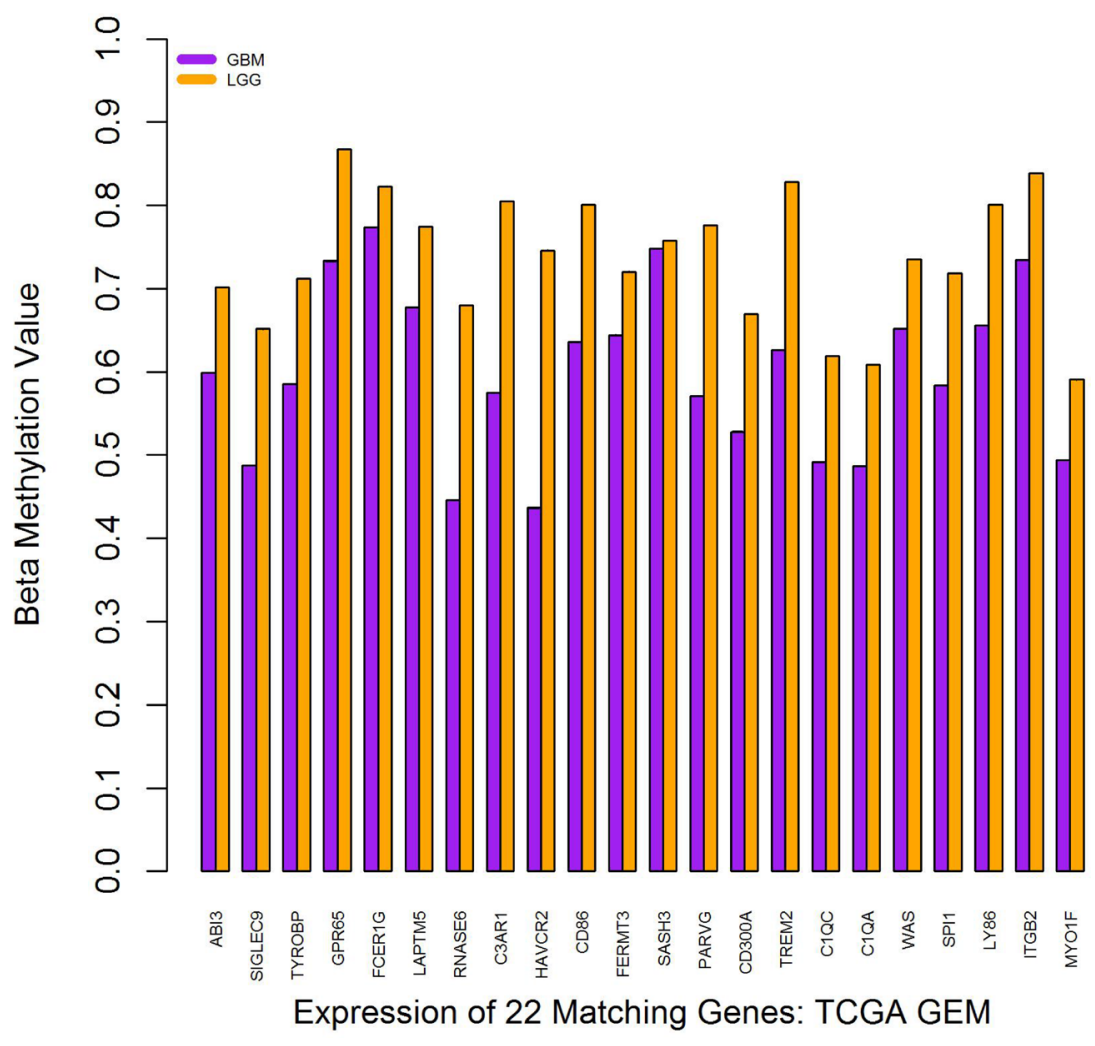

Figure 6: Beta methylation values for the 22 matching glioblastoma genes. Error bars show SEM. Note that, because there are several methylation readings for each dataset and hundreds of datasets per condition, no error bars are visible because the SEM is appreciably zero. 
427 bladder cancer (BLCA) samples, 309 ovarian cancer (OV) samples, and 572 thyroid cancer (THCA) samples. These datasets were compiled into a single GEM, which is an $n \times m$ matrix where $n$ is the number of datasets and $m$ is the number of RNA transcript IDs; each value represents a gene's expression level as quantified by RSEM through the RNASeqV2 workflow $[14,15]$. The TCGA data utilized 73599 knownGene version 5 (kg5) UCSC transcript IDs. As such, the raw GEM was a $73599 \times 2016$ matrix. Outlier expression profiles were detected using a Kolmogorov-Smirnov $(\mathrm{KS})$ test $\left(\mathrm{D}_{\mathrm{N}}>0.15\right)$ implemented in the preprocessCore [16] library. No outliers were detected. All non-zero expression values were log2 transformed and the matrix was quantile normalized.

\section{Brain and random GEM construction}

A brain-specific GEM was constructed using 220 RNAseq datasets from NCBI's SRA database [12]. These 220 samples were the only publiclyavailable samples annotated as brain-specific in the SRA database upon their download on September 16, 2016. These datasets were processed into a GEM using the SRA toolkit v2.5.2 [12], Trimmomatic v0.33 [17], Hisat2-2.0.1-beta [18], Samtools v0.1.19 [19, 20], and Cufflinks v2.2.1 [21]. The Gencode v24 GFF3 file, complete with scaffolds, assembly patches, and alternate loci guided transcript quantification (http://www. gencodegenes.org/releases/24.html). The raw GEM was preprocessed through the methods described above. 16 datasets were removed by the KS test $\left(D_{N}>0.15\right)$, leaving 204 samples in the $\log 2$ normalized GEM. Transcript counts were indexed as 209086 Ensembl hg38 transcript IDs resulted in a $209086 \times 204$ GEM. Of these samples, 38 were GBM tumor samples [9], 138 were normal brain samples [10], and 28 were from Brodmann's Area 9 of Parkinson's Disease patients [11]. A random human GEM was also constructed using 2004 human RNAseq datasets from the NCBI SRA database. These samples were randomly selected from all available paired-end human RNAseq datasets that were produced by an Illumina HiSeq sequencer. This GEM was constructed and preprocessed as described above. The KS test $\left(\mathrm{D}_{\mathrm{N}}>0.15\right)$ removed 211 datasets, resulting in a $209086 \times 1793$ GEM. From this $209086 \times 1793$ preprocessed GEM, 49 transcripts mapped to the genes present in Module 0214 identified in the TCGA Network.

\section{GCN construction and thresholding}

Each normalized GEM was processed with KINC v1.0 [8], a software package that uses Gaussian mixture models (GMMs) before applying pairwise correlation analyses. For each GEM, the OSG-KINC (https://github.com/feltus/ OSG-KINC) workflow was utilized to build a similarity matrix using the KINC software. This workflow utilizes the Pegasus Workflow Management System [22] to execute GMM clustering and pairwise spearman correlation on the

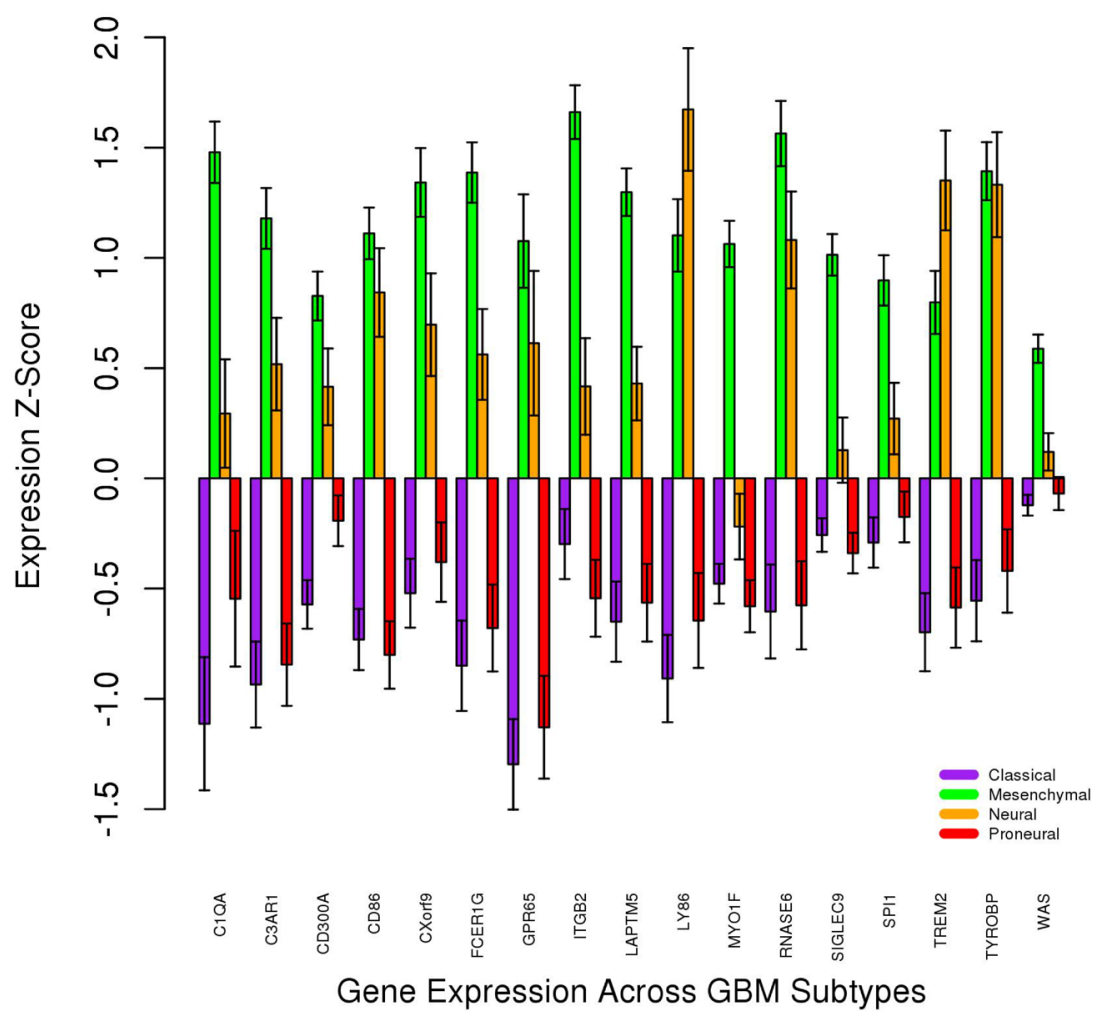

Figure 7: Gene expression levels across GBM subtypes for 17 of the 22 matching genes. Error bars represent SEM. 
Table 4: Mutation enrichment in shared genes

\begin{tabular}{lccc}
\hline Condition & Gene & Direction & Enrichment \\
\hline LGG & ATRX & Co-occurrence & $2.56 \mathrm{E}-06$ \\
LGG & TP53 & Co-occurrence & $5.04 \mathrm{E}-06$ \\
LGG & CIC & Mutual exclusivity & $1.29 \mathrm{E}-03$ \\
LGG & MUC17 & Co-occurrence & 0.0122 \\
LGG & FUBP1 & Mutual exclusivity & 0.0143 \\
LGG & VPS13B & Co-occurrence & 0.019 \\
LGG & MYH8 & Co-occurrence & 0.029 \\
GBM & KCNQ5 & Co-occurrence & $3.84 \mathrm{E}-03$ \\
GBM & MUC17 & Co-occurrence & 0.0126 \\
GBM & LILRB2 & Co-occurrence & 0.016 \\
GBM & PTPRM & Co-occurrence & 0.016 \\
GBM & PIK3R1 & Mutual exclusivity & 0.0326 \\
\hline
\end{tabular}

Open Science Grid (https://www.opensciencegrid.org). By using GMMs prior to each pairwise comparison, KINC [8] samples clusters that result in co-expressed genes. Only clusters spanning 30 or more samples were further processed with Spearman correlations because a Pearson's power analysis found that 30 samples resulted in a false positive rate at $\alpha=0.05$, a false negative at $\beta=0.2$, and an effect size of 0.5. The Brain and TCGA Networks each took about one month to construct with KINC. Globus [24] was used to transfer KINC output files to the Palmetto Cluster at Clemson University (https://www.palmetto.clemson.edu/palmetto/). Random Matrix Thresholding (RMT) [25] was used to find a correlation significance threshold for each similarity matrix produced by KINC. The thresholding process ignored clusters with low expression levels ( $<0.1$ FPKM) and/or less than 30 datasets. Correlations above this experimentally determined significance threshold were extracted for each GCN. The TCGA Network, as described in Ficklin et al. [8], had a correlation threshold of 0.8601 and the Brain Network had a correlation threshold of 0.8801 . Each edge in a GCN represents a relationship between two genes with a correlation value greater than the RMT-defined significance threshold. Link Community Modules (LCM), or groups of co-expressed genes, were identified using the linkcomm R package [13, 26].

\section{TCGA transcript ID mapping}

Biomart (http://useast.ensembl.org/biomart) was used to map each hg38 Ensembl transcript ID in the Brain Network to its corresponding hg38 Associated Gene Name. Using the UCSC hg19 database, the kg5 UCSC transcript IDs in the TCGA Network were mapped to $\mathrm{kg} 6$ and $\mathrm{kg} 7$ UCSC IDs. Using the UCSC hg38 database, these $\mathrm{kg} 7$ UCSC IDs were mapped to $\mathrm{kg} 8$ UCSC IDs, kg9 UCSC IDs, and finally to hg38 Ensembl IDs. Biomart was then used to map each hg38 Ensembl ID to its corresponding Associate Gene Name. 90\% of the original kg5 UCSC IDs mapped to hg38 Ensembl IDs.

\section{GeneFriends co-expression validation}

The 22 matching genes between TCGA M0214 and Brain M0257 were searched as a group in the GeneFriends database [27]. The transcription factor data and the internal co-expression data for the matching genes were downloaded.

\section{Module enrichment analysis}

Each module was tested for sample label enrichment (Fisher's Exact Test $P<0.001$; e.g. LGG, GBM, OV, THCA, BLCA, Normal Brain, and Parkinson's Brain. Only samples present in $>95 \%$ of the edges in a module were considered. Functional term enrichment was performed to associate each module with these functional annotations: Kyoto Encyclopedia of Genes and Genomes (KEGG) [28], Gene Ontology (GO) [29], Reactome [30], InterPro [31], Pfam [32], and Mendelian Inheritance in Man (MIM) [33]. A Fisher's Exact Test $p$-value $<0.001$ was considered significant.

\section{Internetwork comparisons}

For GBM comparisons with the Brain GEM, 63 transcripts in Brain M0257 were used to extract a $63 \times 138$ GEM with only normal brain datasets-no GBM datasets. This mini-GEM was processed with KINC [8] and edges matching those in Brain M0257 were identified. For cancer-specific comparisons with the random GEM, 54 transcripts in TCGA M0214 were identified in the Brain GEM and the Random GEM. 49 of the $54 \mathrm{~kg} 5$ UCSC transcripts in the TCGA GEM mapped to hg38 Ensembl transcripts in the Brain and Random GEMs. These 49 transcripts and their expression values were extracted into a $49 \times 1793$ mini-GEM that was converted into a GCN with KINC. Edges matching those in TCGA M0214 were identified. For the 22 matching genes between 


\begin{tabular}{|c|c|c|}
\hline Term & Term ID & Function \\
\hline $\mathrm{GO}$ & GO:0045087 & $\begin{array}{l}\text { Innate immune responses are defense responses mediated by germline } \\
\text { encoded components that directly recognize components of potential } \\
\text { pathogens. }\end{array}$ \\
\hline $\mathrm{GO}$ & GO:0050776 & $\begin{array}{l}\text { Any process that modulates the frequency, rate or extent of the immune } \\
\text { response, the immunological reaction of an organism to an immunogenic } \\
\text { stimulus. }\end{array}$ \\
\hline $\mathrm{GO}$ & GO:0045650 & $\begin{array}{l}\text { Any process that stops, prevents, or reduces the frequency, rate or extent } \\
\text { of macrophage differentiation. }\end{array}$ \\
\hline $\mathrm{GO}$ & GO:0005581 & $\begin{array}{l}\text { A protein complex consisting of three collagen chains assembled into a } \\
\text { left-handed triple helix. }\end{array}$ \\
\hline $\mathrm{GO}$ & GO:0030853 & $\begin{array}{l}\text { Any process that stops, prevents, or reduces the frequency, rate or extent } \\
\text { of granulocyte differentiation. }\end{array}$ \\
\hline $\mathrm{GO}$ & GO:0006955 & $\begin{array}{l}\text { Any immune system process that functions in the calibrated response of } \\
\text { an organism to a potential internal or invasive threat. }\end{array}$ \\
\hline $\mathrm{GO}$ & GO:0034138 & $\begin{array}{l}\text { Any series of molecular signals generated as a consequence of binding to } \\
\text { toll-like receptor } 3 \text {. }\end{array}$ \\
\hline $\mathrm{GO}$ & GO:0002283 & $\begin{array}{l}\text { The change in morphology and behavior of a neutrophil resulting from } \\
\text { exposure to a cytokine, chemokine, cellular ligand, or soluble factor, } \\
\text { leading to the initiation or perpetuation of an immune response. }\end{array}$ \\
\hline $\mathrm{GO}$ & GO:0002281 & $\begin{array}{l}\text { A change in morphology and behavior of a macrophage resulting from } \\
\text { exposure to a cytokine, chemokine, cellular ligand, or soluble factor, } \\
\text { leading to the initiation or perpetuation of an immune response. }\end{array}$ \\
\hline $\mathrm{GO}$ & GO:0019864 & $\begin{array}{l}\text { Interacting selectively and non-covalently with an immunoglobulin of an } \\
\text { IgG isotype. }\end{array}$ \\
\hline $\mathrm{GO}$ & GO:0071404 & $\begin{array}{l}\text { Any process that results in a change in state or activity of a cell (in terms } \\
\text { of movement, secretion, enzyme production, gene expression, etc.) as a } \\
\text { result of a low-density lipoprotein particle stimulus. }\end{array}$ \\
\hline INTERPRO & IPR001073 & C1q domain \\
\hline INTERPRO & IPR008983 & Tumour necrosis factor-like domain \\
\hline INTERPRO & IPR008160 & Collagen triple helix repeat \\
\hline INTERPRO & IPR013106 & Immunoglobulin V-set domain \\
\hline KEGG & hsa05322 & Systemic lupus erythematosus \\
\hline MIM & 120575 & COMPLEMENT COMPONENT 1, q SUBCOMPONENT, C CHAIN \\
\hline PFAM & PF00386 & $\begin{array}{l}\mathrm{C} 1 \mathrm{q} \text { is a subunit of the } \mathrm{C} 1 \text { enzyme complex that activates the serum } \\
\text { complement system. }\end{array}$ \\
\hline PFAM & PF01391 & Members of this family belong to the collagen superfamily. \\
\hline PFAM & PF07686 & $\begin{array}{l}\text { This domain is found in antibodies as well as neural protein P0 and CTL4 } \\
\text { amongst others. }\end{array}$ \\
\hline REACTOME & R-HSA-173623 & Classical antibody-mediated complement activation \\
\hline REACTOME & R-HSA-198933 & $\begin{array}{l}\text { Immunoregulatory interactions between a Lymphoid and a non-Lymphoid } \\
\text { cell }\end{array}$ \\
\hline REACTOME & R-HSA-166663 & Initial triggering of complement \\
\hline
\end{tabular}

TCGA M0214 and Brain M0257, the normalized GBM and LGG expression values in the TCGA GEM were compared with a Student's $T$-Test. The GBM and normal brain expression values in the Brain GEM were also compared with a Student's T-Test. The Matlab clustergram function was used to generate heatmaps that represent gene expression intensities. We recognized that the Brain GEM, created from various publicly available RNAseq 
datasets, required further normalization within conditions; unlike the TCGA datasets, the Brain datasets were not all derived by the same research network using the same RNASeqV2 workflow. Thus, GBM expression values of the 22 matching genes were divided by the GBM global median expression value in the Brain GEM (1.1398). Similarly, the normal brain expression values of the 22 matching genes were divided by the normal brain global median expression value in the Brain GEM (1.7215). A Student's $T$-Test was also used to compare ELF1 and NF1 expression levels across different conditions in the TCGA and Brain GEMs.

\section{Module DNA methylation analysis}

All publicly available beta methylation datasets for GBM and LGG were downloaded from the Genomic Data Commons' TCGA Data Portal (https://gdc-portal.nci.nih. gov/) on January 27, 2017. 1607 datasets, including 534 LGG and 450 GBM datasets, were downloaded. The beta methylation values for the 22 matching genes across each cancer were compared with a Student's $T$-Test.

\section{External evidence for internetwork module relationships}

The 22 genes matching between TCGA M0214 and Brain M0257 were queried in the Gene Set Enrichment Analysis database [34] and the resulting transcription factor data was downloaded. These 22 genes were also provided to the RegNetwork database [35] to find possible regulatory mechanisms. In addition, RNase6, one of the 22 matching genes, was searched in the ImmuNet database [36] to find functional similarities to genes in the complement cascade. The genes with ImmuNet confidence levels $>0.99$ were downloaded. The gbmSygnal Network [37] was also queried and 16 biclusters with three or more of the 22 matching genes were discovered. The 22 matching genes were also provided to the Glioblastoma Bio Discovery Portal [38] using the "Verhaak Core" participants option and the "3-Platform Aggregates" experiment option. Finally, cBioPortal [39, 40] was used to analyze the 22 matching genes in patient samples; the 22 matching genes were queried twice; first, as a gene set using LGG ( $n=283$ tumors) data, and second, using GBM ( $n=136$ tumors) data from the "TCGA, Provisional" dataset. cBioPortal uses a Student's $T$-Test for its reverse phase protein array comparison and a Fisher Exact Test for mutation enrichment.

\section{Abbreviations}

GBM: glioblastoma, also known as glioblastoma multiforme; LGG: lower grade glioma; BLCA: bladder urothelial carcinoma; THCA: thyroid carcinoma; OV: ovarian serous cystadenocarcinoma; GEM: gene expression matrix; GCN: gene co-expression network;
TCGA: the Cancer Genome Atlas; KINC: KnowledgeIndependent Network Construction; kg: knownGene; OSG: Open Science Grid; GMM: Gaussian mixture model; RMT: Random Matrix Thresholding; LCM: Link Community Module.

\section{Author contributions}

Project Design: LJD, FAF; Data Acquisition: LJD; KINC Software Development: SPF, FAF; GEM/KINC Workflow Design: LJD, WLP; Manuscript Writing: LJD, WLP, SPF, FAF.

\section{ACKNOWLEDGMENTS}

This work used Clemson University's Palmetto Cluster, Washington State University's Kamiak Cluster (both high performance compute clusters) and the Open Science Grid (OSG). The OSG is supported by the National Science Foundation and the U.S. Department of Energy's Office of Science. We acknowledge the assistance of M. Rynge, D. Balamurugan, and the OSG support staff for technical support and assistance. We acknowledge the assistance of J. Schipper from Van Andel Institute for reviewing the manuscript.

\section{CONFLICTS OF INTEREST}

The authors declare no conflicts of interest.

\section{REFERENCES}

1. American Brain Tumor Association. Glioblastoma. http:// www.abta.org/brain-tumor-information/types-of-tumors/gli oblastoma.html. 2017.

2. Nelson JS, Burchfiel CM, Fekedulegn D, Andrew ME. Potential risk factors for incident glioblastoma multiforme: the Honolulu Heart Program and Honolulu-Asia Aging Study. J Neurooncol. 2012; 109:315-21. https://doi. org/10.1007/s11060-012-0895-3.

3. Dunn GP, Andronesi OC, Cahill DP. From genomics to the clinic: biological and translational insights of mutant IDH1/2 in glioma. Neurosurg Focus. 2013; 34:E2. https:// doi.org/10.3171/2012.12.focus12355.

4. Liu KW, Hu B, Cheng SY. Platelet-derived growth factor receptor alpha in glioma: a bad seed. Chin J Cancer. 2011; 30:590-602. https://doi.org/10.5732/cjc.011.10236.

5. Petronis A. Epigenetics as a unifying principle in the aetiology of complex traits and diseases. Nature. 2010; 465:721-7. https://doi.org/10.1038/nature09230.

6. Schuster SC. Next-generation sequencing transforms today's biology. Nat Methods. 2008; 5:16-8. https://doi. org/10.1038/nmeth1156.

7. Weinstein JN, Collisson EA, Mills GB, Shaw KR, Ozenberger BA, Ellrott K, Shmulevich I, Sander C, Stuart 
JM. The Cancer Genome Atlas Pan-Cancer analysis project. Nat Genet. 2013; 45:1113-20. https://doi.org/10.1038/ ng.2764.

8. Ficklin SP, Dunwoodie LJ, Poehlman WL, Watson C, Roche KE, Feltus FA. Discovering Condition-Specific Gene Co-Expression Patterns Using Gaussian Mixture Models: A Cancer Case Study. Sci Rep. 2017; 7:8617. https://doi. org/10.1038/s41598-017-09094-4.

9. Gabrusiewicz K, Rodriguez B, Wei J, Hashimoto Y, Healy LM, Maiti SN, Thomas G, Zhou S, Wang Q, Elakkad A, Liebelt BD, Yaghi NK, Ezhilarasan R, et al. Glioblastomainfiltrated innate immune cells resemble M0 macrophage phenotype. JCI Insight. 2016; 1. https://doi.org/10.1172/jci. insight. 85841 .

10. Hwang T, Park CK, Leung AK, Gao Y, Hyde TM, Kleinman JE, Rajpurohit A, Tao R, Shin JH, Weinberger DR. Dynamic regulation of RNA editing in human brain development and disease. Nat Neurosci. 2016; 19:1093-9. https://doi. org/10.1038/nn.4337.

11. Dumitriu A, Golji J, Labadorf AT, Gao B, Beach TG, Myers RH, Longo KA, Latourelle JC. Integrative analyses of proteomics and RNA transcriptomics implicate mitochondrial processes, protein folding pathways and GWAS loci in Parkinson disease. BMC Medical Genomics. 2016; 9:5. https://doi.org/10.1186/s12920-016-0164-y.

12. Leinonen R, Sugawara H, Shumway M. The sequence read archive. Nucleic Acids Res. 2011; 39:D19-21. https://doi. org/10.1093/nar/gkq1019.

13. Ahn YY, Bagrow JP, Lehmann S. Link communities reveal multiscale complexity in networks. Nature. 2010; 466: 761-4. https://doi.org/10.1038/nature09182.

14. Wang K, Singh D, Zeng Z, Coleman SJ, Huang Y, Savich GL, He X, Mieczkowski P, Grimm SA, Perou CM, MacLeod JN, Chiang DY, Prins JF, et al. MapSplice: accurate mapping of RNA-seq reads for splice junction discovery. Nucleic Acids Res. 2010; 38:e178. https://doi. org/10.1093/nar/gkq622.

15. Pihl T. (2013). RNASeq Version 2.

16. Bolstad BM. preprocessCore: A collection of pre-processing functions. R package version. 2013; 1.

17. Bolger AM, Lohse M, Usadel B. Trimmomatic: a flexible trimmer for Illumina sequence data. Bioinformatics. 2014; 30:2114-20. https://doi.org/10.1093/bioinformatics/btu170.

18. Kim D, Langmead B, Salzberg SL. HISAT: a fast spliced aligner with low memory requirements. Nat Methods. 2015; 12:357-60. https://doi.org/10.1038/nmeth.3317.

19. Li H. A statistical framework for SNP calling, mutation discovery, association mapping and population genetical parameter estimation from sequencing data. Bioinformatics. 2011; 27:2987-93. https://doi.org/10.1093/bioinformatics/ btr509.

20. Li H, Handsaker B, Wysoker A, Fennell T, Ruan J, Homer N, Marth G, Abecasis G, Durbin R. The Sequence Alignment/Map format and SAMtools. Bioinformatics.
2009; 25:2078-9. https://doi.org/10.1093/bioinformatics/ btp352.

21. Trapnell C, Williams BA, Pertea G, Mortazavi A, Kwan G, van Baren MJ, Salzberg SL, Wold BJ, Pachter L. Transcript assembly and abundance estimation from RNA-Seq reveals thousands of new transcripts and switching among isoforms. Nature biotechnology. 2010; 28:511-5. https:// doi.org/10.1038/nbt.1621.

22. Deelman E, Vahi K, Juve G, Rynge M, Callaghan S, Maechling PJ, Mayani R, Chen W, Ferreira da Silva R, Livny M, Wenger K. Pegasus, a workflow management system for science automation. Future Generation Computer Systems. 2015; 46:17-35. https://doi.org/http://dx.doi. org/10.1016/j.future.2014.10.008.

23. Anderson AC. Tim-3: an emerging target in the cancer immunotherapy landscape. Cancer Immunol Res. 2014; 2:393-8. https://doi.org/10.1158/2326-6066.cir-14-0039.

24. Foster I. Globus Toolkit Version 4: Software for ServiceOriented Systems. IFIP International Federation for Information Processing. 2005:2-13.

25. Luo F, Yang Y, Zhong J, Gao H, Khan L, Thompson DK, Zhou J. Constructing gene co-expression networks and predicting functions of unknown genes by random matrix theory. BMC Bioinformatics. 2007; 8:299. https://doi. org/10.1186/1471-2105-8-299.

26. Kalinka AT, Tomancak P. linkcomm: an R package for the generation, visualization, and analysis of link communities in networks of arbitrary size and type. Bioinformatics. 2011; 27:2011-2. https://doi.org/10.1093/bioinformatics/btr311.

27. van Dam S, Craig T, de Magalhaes JP. GeneFriends: a human RNA-seq-based gene and transcript co-expression database. Nucleic Acids Res. 2015; 43:D1124-32. https:// doi.org/10.1093/nar/gku1042.

28. Kanehisa M, Goto S. KEGG: kyoto encyclopedia of genes and genomes. Nucleic Acids Res. 2000; 28:27-30.

29. Ashburner M, Ball CA, Blake JA, Botstein D, Butler H, Cherry JM, Davis AP, Dolinski K, Dwight SS, Eppig JT, Harris MA, Hill DP, Issel-Tarver L, et al. Gene ontology: tool for the unification of biology. The Gene Ontology Consortium. Nat Genet. 2000; 25:25-9. https://doi. org/10.1038/75556.

30. Fabregat A, Sidiropoulos K, Garapati P, Gillespie M, Hausmann K, Haw R, Jassal B, Jupe S, Korninger F, McKay S, Matthews L, May B, Milacic M, et al. The Reactome pathway Knowledgebase. Nucleic Acids Res. 2016; 44:D481-7. https://doi.org/10.1093/nar/gkv1351.

31. Finn RD, Attwood TK, Babbitt PC, Bateman A, Bork P, Bridge AJ, Chang HY, Dosztanyi Z, El-Gebali S, Fraser M, Gough J, Haft D, Holliday GL, et al. InterPro in 2017-beyond protein family and domain annotations. Nucleic Acids Res. 2017; 45:D190-d9. https://doi.org/10.1093/nar/gkw1107.

32. Finn RD, Coggill P, Eberhardt RY, Eddy SR, Mistry J, Mitchell AL, Potter SC, Punta M, Qureshi M, SangradorVegas A, Salazar GA, Tate J, Bateman A. The Pfam 
protein families database: towards a more sustainable future. Nucleic Acids Res. 2016; 44:D279-85. https://doi. org/10.1093/nar/gkv1344.

33. Amberger JS, Bocchini CA, Schiettecatte F, Scott AF, Hamosh A. OMIM.org: Online Mendelian Inheritance in Man (OMIM(R)), an online catalog of human genes and genetic disorders. Nucleic Acids Res. 2015; 43:D789-98. https://doi.org/10.1093/nar/gku1205.

34. Subramanian A, Tamayo P, Mootha VK, Mukherjee S, Ebert BL, Gillette MA, Paulovich A, Pomeroy SL, Golub TR, Lander ES, Mesirov JP. Gene set enrichment analysis: a knowledge-based approach for interpreting genomewide expression profiles. Proc Natl Acad Sci U S A. 2005; 102:15545-50. https://doi.org/10.1073/pnas.0506580102.

35. Liu ZP, Wu C, Miao H, Wu H. RegNetwork: an integrated database of transcriptional and post-transcriptional regulatory networks in human and mouse. Database (Oxford). 2015; 2015. https://doi.org/10.1093/database/ bav095.

36. Gorenshteyn D, Zaslavsky E, Fribourg M, Park CY, Wong AK, Tadych A, Hartmann BM, Albrecht RA, Garcia-Sastre A, Kleinstein SH, Troyanskaya OG, Sealfon SC. Interactive Big Data Resource to Elucidate Human Immune Pathways and Diseases. Immunity. 2015; 43:605-14. https://doi. org/10.1016/j.immuni.2015.08.014.

37. Plaisier CL, O’Brien S, Bernard B, Reynolds S, Simon Z, Toledo CM, Ding Y, Reiss DJ, Paddison PJ, Baliga NS. Causal Mechanistic Regulatory Network for Glioblastoma Deciphered Using Systems Genetics Network Analysis. Cell Systems. 2016; 3:172-86. https://doi.org/10.1016/j. cels.2016.06.006.

38. Celiku O, Johnson S, Zhao S, Camphausen K, Shankavaram U. Visualizing molecular profiles of glioblastoma with GBM-BioDP. PLoS One. 2014; 9:e101239. https://doi. org/10.1371/journal.pone.0101239.

39. Gao J, Aksoy BA, Dogrusoz U, Dresdner G, Gross B, Sumer SO, Sun Y, Jacobsen A, Sinha R, Larsson E, Cerami E, Sander C, Schultz N. Integrative analysis of complex cancer genomics and clinical profiles using the cBioPortal. Sci Signal. 2013; 6:pl1. https://doi.org/10.1126/ scisignal.2004088.

40. Cerami E, Gao J, Dogrusoz U, Gross BE, Sumer SO, Aksoy BA, Jacobsen A, Byrne CJ, Heuer ML, Larsson E, Antipin Y, Reva B, Goldberg AP, et al. The cBio Cancer Genomics Portal: An Open Platform for Exploring Multidimensional Cancer Genomics Data. Cancer Discovery. 2012; 2:401-4. https://doi.org/10.1158/2159-8290.cd-12-0095.
41. Shannon P, Markiel A, Ozier O, Baliga NS, Wang JT, Ramage D, Amin N, Schwikowski B, Ideker T. Cytoscape: a software environment for integrated models of biomolecular interaction networks. Genome Res. 2003; 13:2498-504. https://doi.org/10.1101/gr.1239303.

42. Pakneshan S, Safarpour D, Tavassoli F, Jabbari B. Brain metastasis from ovarian cancer: a systematic review. J Neurooncol. 2014; 119:1-6. https://doi.org/10.1007/ s11060-014-1447-9.

43. Kommoss S, Winterhoff B, Oberg A, Konecny GE, Wang C, Riska SM, Fan JB, Maurer MJ, April C, Shridhar V, Kommoss F, du Bois A, Hilpert F, et al. Bevacizumab may differentially improve ovarian cancer outcome in patients with proliferative and mesenchymal molecular subtypes. Clinical Cancer Research. 2017. https://doi. org/10.1158/1078-0432.ccr-16-2196.

44. Verhaak RG, Hoadley KA, Purdom E, Wang V, Qi Y, Wilkerson MD, Miller CR, Ding L, Golub T, Mesirov JP, Alexe G, Lawrence M, O’Kelly M, et al. An integrated genomic analysis identifies clinically relevant subtypes of glioblastoma characterized by abnormalities in PDGFRA, IDH1, EGFR and NF1. Cancer cell. 2010; 17:98. https:// doi.org/10.1016/j.ccr.2009.12.020.

45. Weber GL, Parat MO, Binder ZA, Gallia GL, Riggins GJ. Abrogation of PIK3CA or PIK3R1 reduces proliferation, migration, and invasion in glioblastoma multiforme cells. Oncotarget. 2011; 2:833-49.

46. Feng X, Tonnesen MG, Peerschke EI, Ghebrehiwet B. Cooperation of $\mathrm{C} 1 \mathrm{q}$ receptors and integrins in $\mathrm{C} 1 \mathrm{q}-$ mediated endothelial cell adhesion and spreading. J Immunol. 2002; 168:2441-8.

47. Shinjyo N, Stahlberg A, Dragunow M, Pekny M, Pekna M. Complement-derived anaphylatoxin $\mathrm{C} 3$ a regulates in vitro differentiation and migration of neural progenitor cells. Stem Cells. 2009; 27:2824-32. https://doi.org/10.1002/ stem.225.

48. Bulla R, Tripodo C, Rami D, Ling GS, Agostinis C, Guarnotta C, Zorzet S, Durigutto P, Botto M, Tedesco F. $\mathrm{C} 1 \mathrm{q}$ acts in the tumour microenvironment as a cancerpromoting factor independently of complement activation. Nat Commun. 2016; 7:10346. https://doi.org/10.1038/ ncomms 10346.

49. Carro MS, Lim WK, Alvarez MJ, Bollo RJ, Zhao X, Snyder EY, Sulman EP, Anne SL, Doetsch F, Colman H, Lasorella A, Aldape K, Califano A, et al. The transcriptional network for mesenchymal transformation of brain tumours. Nature. 2010; 463:318-25. https://doi.org/10.1038/nature08712. 